\title{
THE SPEAR 3 DIAGNOSTIC BEAMLINES
}

\author{
J. Corbett, C. Limborg-Deprey, W. Mok and A. Ringwall, SLAC, Menlo Park, California
}

\section{Abstract}

SPEAR 3 has two diagnostic beam lines: an x-ray pinhole camera and a visible/UV laboratory. The pinhole camera images $\sim 8 \mathrm{keV}$ dipole radiation on a phosphor screen with a remote computer to capture digital images and a parallel video signal to the control room. The visible/UV beam line features an $8 \mathrm{~mm}$ high GlidCop 'cold finger' to remove the x-ray core of the beam. The remaining light is deflected horizontally onto an optical bench where it is focused via reflective (Cassegrain) or refractive optics. The visible beam can be split into branch lines for a variety of experimental applications. This paper describes the experimental set up and projected use of both systems.

\section{INTRODUCTION}

Synchrotron radiation is perhaps the most direct means to measure charge distribution in electron storage rings [1-3]. Due to the relatively short pulse lengths and diffraction effects, however, the measurements can be challenging and require signal deconvolution to extract the desired information. For emittance measurements, $x$ ray pinhole cameras [4] were the instrument of choice until the emergence of the 2-slit optical interferometer [5]. Direct imaging in the visible regime is also possible with modern equipment [6]. Optical imaging also has application the study of transverse beam dynamics. In the longitudinal direction, speed is of essence so fast-gated cameras, steak cameras and photodiodes find application.

For SPEAR 3, the existing pinhole camera has been used for emittance measurements [7]. An additional visible/UV dipole beam line was constructed to study both longitudinal and transverse beam properties [8]. The new beam line will feature a reflective (Cassegrain) telescope feeding parallel optical beam lines with a $2 \mathrm{nS}$ gated camera [9], a streak camera and auxiliary branch lines for photodiode measurements, photomultipliers, position monitors, video monitoring, etc. In this paper, we outline features of and plans for both the x-ray pinhole camera and visible/UV beam line.

Table 1: Pinhole Camera Source (1\% coupling)

\begin{tabular}{|c|c|c|}
\hline$\beta_{\mathrm{x} / \mathrm{y},} \alpha_{\mathrm{x} / \mathrm{y}}$ & $0.80 / 13.75$ & $-0.414 / 2.758$ \\
\hline$\eta_{\mathrm{x} / \mathrm{y},} \eta_{\mathrm{x} / \mathrm{y}}$ & $0.106 / 0.0$ & $0.174 / 0.0$ \\
\hline $\begin{array}{c}\varepsilon_{\mathrm{x} / \mathrm{y}}, \sigma_{\mathrm{x} / \mathrm{y}} \\
(\mathrm{dp} / \mathrm{p}=0.1 \%)\end{array}$ & $18.6 / 0.186$ & $160 / 50 \mu \mathrm{m}$ \\
\hline
\end{tabular}

* This work was supported U.S. Department of Energy under Contract Number DE-AC02-76SF00515.

\section{PINHOLE CAMERA}

Pinhole cameras provide a well known means to image synchrotron radiation in the $x$-ray regime. The SPEAR 3 system images dipole radiation with geometric camera distances $\mathrm{d}_{1}=10 \mathrm{~m}, \mathrm{~d}_{2}=5.87 \mathrm{~m}(\mathrm{M}=0.59)$. Dating from earlier applications, the pinhole camera was refurbished in 1999 and successfully used to reduce emittance coupling in SPEAR 2 from over 3\% to less than 1\% [7]. The remote-controlled pinhole aperture $(30 \mu \mathrm{m}$ × $25 \mu \mathrm{m}$ Ta blades) and camera components (YAG phosphor, Si mirror, Pulnix TM1300 CCD camera) were modeled after the NSLS design [10]. Beam size was measured by deconvolving the (Fraunhoffer) point-spread function for pinhole diffraction and the detector resolution in reciprocal space. Details of the camera system can be found in [7].
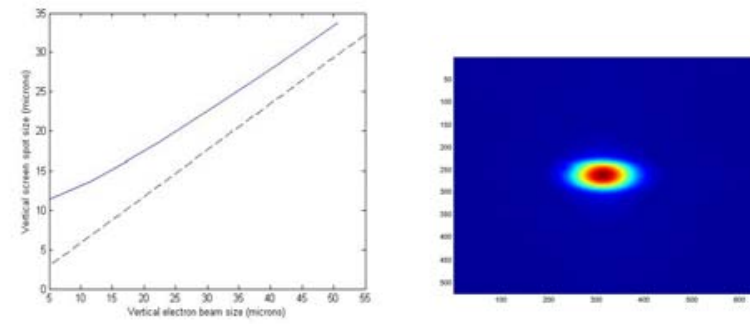

Figure 1: Fresnel calculation of vertical spot size on screen as a function of vertical source size. The dashed line represents the geometric approximation. Figure to right shows a typical camera image.

For SPEAR 3, the pinhole heat sink was upgraded and a remote PC with frame-grabber was moved into the new beam line hutch. Beam size measurements, however, became increasingly difficult: in SPEAR 3 the dipole source was reduced by about a factor of 4 in both planes ( $1 \%$ coupling). Furthermore, using response matrix analysis techniques, the emittance coupling was reduced to $\sim 0.05 \%$ or a vertical beam size of $\sigma_{\mathrm{y}} \sim 10 \mu \mathrm{m}$. To compute Fresnel diffraction effects, we utilize the integration program of J. Bergstrom at the CLS. Figure 1 illustrates a calculation of the diffracted image size as a function of the electron beam source size in the vertical plane. A bi-Gaussian fit is performed on the digitized image and the source size is read directly from the plot. Preliminary measurements indicate a coupling coefficient of $\kappa \sim 0.5 \%$, well above the predicted value of $\sim 0.05 \%$. In the horizontal plane, $\varepsilon_{\mathrm{x}}=16-18 \mathrm{~nm}-\mathrm{rad}$, in agreement with theory. 


\section{Plans for the Pinhole Camera}

Although the pinhole camera is operationally robust, the system needs upgrades to increase resolution. Plans include installation of a $4.65 \mu \mathrm{m}$ pixel GreyPoint CCD camera [11], re-calibration of the pixel $/ \mu \mathrm{m}$ conversion factor and improvements to the Gaussian beam fitting routines. Further analysis and optimization of the x-ray beam spectral transmission is required. The results will be coupled with standard phase-space calculations including geometric acceptance considerations and integrated into the Fresnel diffraction routine.

In conjunction with the optical beam line and beam scraper systems, the pinhole camera will be used for coupling and lifetime studies as a function of single bunch current, total beam current, coupling parameters, accelerating voltage and ID configuration. Correlation with knife-edge studies on other SPEAR 3 beam lines can be useful for system calibration. The real-time image will be analyzed for beam size and time-dependent effects.

\section{OPTICAL LIGHT MONITOR}

SPEAR 3 was constructed to function as a high-current, low impedance storage ring. The unique copper vacuum chamber design, copper-plated ID chambers, modedamped RF cavities, stripline injection kickers and lowimpedance bellows push the zero-chromaticity instability thresholds out to approximately $250 \mathrm{~mA}$. The SPEAR 3 bunch spacing is $\sim 2 \mathrm{nS}(476.31 \mathrm{MHz})$ and the bunch length is $16 \mathrm{pS}$, so the $2 \mathrm{nS}$ gated camera can resolve individual bunches but sub-nanosecond equipment (e.g. streak camera) will be necessary to reliably measure bunch length and bunch structure.

To date, stable beam has been observed with up to 200 $\mathrm{mA}$ circulating current. In the near future, the SPEAR 3 program calls for operating currents up to $500 \mathrm{~mA}$ and high charge-density timing mode operation as well as topup injection. We anticipate the optical diagnostic laboratory will play an important role in the observation, analysis and control of beam instabilities as they arise.

The layout of the optical beam line begins with a $3.5 \times 6$ mrad rectangular aperture followed a horizontal 'cold finger' that intercepts the hot X-ray core of the beam at 7.5 $\mathrm{m}$ from the source $(+/-0.6 \mathrm{mrad})$. The remaining visible/UV beam is reflected 18 degrees horizontally by a $\mathrm{Rh}$-coated silicon mirror. The mirror has remote pitch control but the cold finger is stationary. Photons up to approximately $8 \mathrm{eV}$ with both polarizations propagate invacuum through a shielding penetration onto a $3 \mathrm{~m}^{2}$ optical bench.

Table 2: Optical monitor source (1\% coupling) Source is $14 \%$ into first dipole on Girder 3

\begin{tabular}{|c|c|c|}
\hline$\beta_{\mathrm{x} / \mathrm{y},} \alpha_{\mathrm{x} / \mathrm{y}}$ & $1.86 / 14.38$ & $1.523 /-2.23$ \\
\hline$\eta_{\mathrm{x} / \mathrm{y},} \eta_{\mathrm{x}}{ }^{\prime} / \mathrm{y}$ & $0.003 / 0.0$ & $0.026 / 0.0$ \\
\hline $\begin{array}{c}\varepsilon_{\mathrm{x} / \mathrm{y}}, \sigma_{\mathrm{x} / \mathrm{y}} \\
(\mathrm{dp} / \mathrm{p}=0.1 \%)\end{array}$ & $18.6 / 0.186$ & $182 / 50.8 \mu \mathrm{m}$ \\
\hline
\end{tabular}

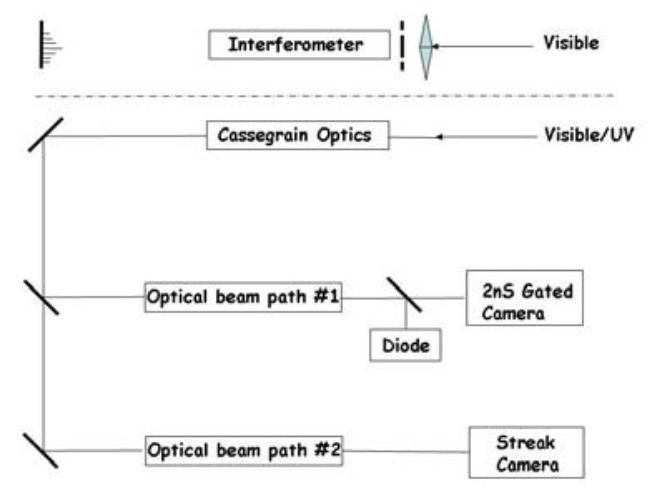

Figure 2: Single optical beam path for interferometer configuration (top) and dual optical beam path configuration (bottom). Reflective Cassegrain optics permits UV imaging on the CCD for improved resolution.

Since the light monitor is directly downstream from the septum and the beam line is open under injection conditions, permanent magnets are used to sweep out stray electrons. A Pb-lined 'hutch' with He-atmosphere and two near-normal reflecting mirrors rejects stray highenergy photons.

Once on the optical bench, the $\sim 10 \mathrm{~cm}$ diameter beam can be focused with either the $2 \mathrm{~m}$ Cassegrain telescope [8] or a $150 \mathrm{~mm}$ diameter achromatic lens. The Cassegrain system permits operation in the near-UV (40 $\mu \mathrm{m}$ resolution at $\lambda=250 \mathrm{~nm}$ ) while the refractive optics has the advantage of operational simplicity and the possibility for interferometric analysis of the vertical beam size. The visible beam can be imaged with either system in a straight, inline configuration, or as illustrated in Fig. 2, folded back and split into multiple optical beam paths with appropriate relay optics. Parallel ray optics in the beam lines is optimum for insertion of filters, slits, and polarizers [12].

\section{Plans for the Optical Light Monitor}

First light into the optical laboratory is expected in late 2005. The initial configuration will block UV light and utilize the $150 \mathrm{~mm}$ refractive lens with dual optical beam paths. One beam path will be equipped with the $2 \mathrm{nS}$ gated camera [9] to image the transverse beam profile under a variety of conditions:

- Injection

- Variable single-bunch and total beam current

- Variable coupling conditions

- Onset of beam instability

Along the line of work performed at the KEK-PF, optical branch lines could be utilized for transient bunchby-bunch diagnostics based on a high-speed shutter or longitudinal bunch-structure measurements [13, 14]. A video camera will monitor the beam in the control room. 
Referring to the top of Fig. 2, the refractive lens can also be configured as part of an optical interferometer. In this mode, the visible light is imaged straight ahead on the CCD camera after passing through a variable spacing double-slit. Mitsuhashi reports measurement resolutions approaching $1 \mu \mathrm{m}$ [5]. Fisher has constructed a similar device at SLAC for PEP-II [15].

The second optical beam path is available for streak camera measurements. Here's where it gets interesting - a sub-nanosecond streak camera can measure a range of single- and multi-bunch beam dynamic features in both the longitudinal and transverse and planes [16].

Examples include:

- Bunch length monitor/broadband impedance

- Longitudinal single-bunch profile measurements

- Microwave instability threshold

- Coupled-bunch instability (longitudinal, transverse)

\section{SUMMARY}

The SPEAR 3 pinhole camera has been commissioned yet requires component upgrades and software refinement to accurately image vertical beam sizes approaching 10 $\mu \mathrm{m}$. The optical beam line can accept photons into the near UV region and is scheduled to open at the end of 2005. This beam line will initially be equipped with refractive optics to image visible light on a fast-gated camera. Build-out of the beam line includes photodiode diagnostics, an optical interferometer, and streak camera to study impedance and single- and multi-bunch beam dynamics as the beam current is raised to $500 \mathrm{~mA}$ and additional small-gap ID's are installed.

\section{ACKNOWLEDGEMENTS}

The authors would like to thank the SSRL technicians and summer students who have contributed to the x-ray pinhole and synchrotron light monitor projects. The ongoing collaboration with J. Bergstrom is gratefully acknowledged.

\section{REFERENCES}

[1] A. Hofmann and F. Meot, "Optical Resolution of Beam Cross-Section Measurements by Means of Synchrotron Radiation", NIM 203, 1982, p. 483.

[2] H. Wiedemann, "Synchrotron Radiation", SpringerVerlag, Berlin, 2003.

[3] A. Hofmann, "The Physics of Synchrotron Radiation", Cambridge University Press, 2004.

[4] P. Elleaume et al., "Measuring Beam Sizes and Ultra-Small Electron Emittances Using an X-ray Pinhole Camera," J. of Synch. Rad. 2, 1995, p. 1774.

[5] T. Mitsuhashi, "Measurement of Small Transverse Beam Size Using Interferometry," DIPAC 2001, Grenoble, France, p. 26.

[6] A. Andersson, "Electron beam profile measurements and emittance manipulation at the MAX-laboratory, PhD Thesis, Lund, 1997.

[7] C. Limborg et al., "A Pinhole Camera for SPEAR 2," EPAC 2000, Vienna, p. 1774.

[8] C. Limborg et al., "An Ultra-Violet Light Monitor for SPEAR 3,” EPAC 2002, Paris, p. 1924.

[9] Roper Scientific, PiMax Camera, UV enhanced, www.princetoninstruments.com.

[10] J. Safranek and P. Stefan, "Emittance Measurement at the NSLS X-Ray Ring," EPAC 1996,

[11] www.GreyPoint.com (Flea model).

[12] J. Bergstrom, private communication.

[13] A. Mochihashi, "Observation of Transverse Instability Using Bunch-by-Bunch Beam Diagnostic System in KEK-PF," EPAC 2000.

[14] M. Tobiyama et al, "Measurement of Bunch TimeStructure in KEK PF," PAC 2003, p. 2409.

[15] A. Fisher, "A Synchrotron-Light Interferometer for PEP-II," BIW 2000, Cambridge, MA.

[16] K. Scheidt, "Review of Streak Cameras for Accelerators: Features, Applications and Results," EPAC 2000, Vienna, p. 182. 